\title{
Divided by an ocean of water but united in an ocean of uncertainty: A transatlantic review of mesothelioma surgery guidelines
}

\author{
David A. Waller, ${ }^{\mathrm{a}}$ Isabelle Opitz, ${ }^{\mathrm{b}}$ Raphael Bueno, ${ }^{\mathrm{c}}$ Paul Van Schil, ${ }^{\mathrm{d}}$ Giuseppe Cardillo, ${ }^{\mathrm{e}}$ David Harpole, ${ }^{\mathrm{f}}$ \\ Prasad S. Adusumilli, ${ }^{\mathrm{g}}$ and Marc De Perrot ${ }^{\mathrm{h}}$
}

The recently published European guidelines on the management of malignant pleural mesothelioma (MPM) ${ }^{1}$ allows us to compare its surgical content with that contained in the American Society of Clinical Oncology (ASCO) guidelines published 2 years ago. ${ }^{2}$ Both were extensive and thorough guidelines focusing on many aspect of MPM. In this editorial, we will point out the many similarities, but also many subtle differences which bear further consideration, particularly in terms of radical surgery for MPM. Moreover, there remain many areas of clinical uncertainty common to both sides that should direct future research.

There is agreement that surgeons have an important role in making an accurate diagnosis by obtaining sufficient numbers of large and deep pleural biopsies (by either video-assisted thoracoscopy or by mini-thoracotomy in

From aBarts Thorax Centre, St Bartholomew's Hospital, London, United Kingdom; ${ }^{b}$ Department of Thoracic Surgery, University Hospital Zurich, Zurich, Switzerland; ' Division of Thoracic Surgery, Brigham and Women's Hospital/Harvard Medical School, Boston, Mass; ${ }^{\mathrm{d}}$ Department of Thoracic and Vascular Surgery, Antwerp University Hospital/Antwerp University, Antwerp, Belgium; e Department of Thoracic Surgery, Azienda Ospedaliera San Camillo Forlanini, Rome, Italy; ${ }_{\mathrm{f}}^{\mathrm{D}}$ Department of Surgery, Duke University Medical Center, Durham, NC; ${ }^{g}$ Memorial Sloan Kettering Cancer Center, New York, NY; ${ }^{\mathrm{h}}$ Division of Thoracic Surgery, Department of Surgery, University of Toronto, Toronto General Hospital, Toronto, Ontario, Canada; and ${ }^{\mathrm{i}}$ Division of Thoracic Surgery, Department of Immunology, University of Toronto, Toronto General Hospital, Toronto, Ontario, Canada.

This editorial has been co-published with permission in The Journal of Thoracic and Cardiovascular Surgery, European Journal of Cardio-Thoracic Surgery, and The Annals of Thoracic Surgery.

Cite this article as: Waller DA, Opitz I, Bueno R, Van Schil P, Cardillo G, Harpole D, et al. Divided by an ocean of water but united in an ocean of uncertainty: a transatlantic review of mesothelioma surgery guidelines. $J$ Thorac Cardiovasc Surg. 2021;161:1922-5.

Disclosures: Raphael Bueno: Research grants and clinical trials support from MedGenome, Roche, Verastem, Genentech, Merck, Gritstone, Epizyme, Siemens, Celsius, and NIH. In addition, Raphael Bueno has 4 patents through the Brigham and Women's Hospital and Equity in a new start-up company, Navigation Sciences. Paul Van Schil: external expert Merck Sharp Dome, AstraZeneca, and Institut National du Cancer, France (institutional fees only). Marc De Perrot: External expert AstraZeneca and Bayer. All other authors reported no conflicts of interest.

Address for reprints: David A. Waller, Barts Thorax Centre, St Bartholomew's Hospital, West Smithfield, London EC1A 7BE, United Kingdom (E-mail: david. waller1@nhs.net).

J Thorac Cardiovasc Surg 2021;161:1922-5

$0022-5223 / \$ 36.00$

Copyright (C) 2020 Jointly between The American Association for Thoracic Surgery, the European Association for Cardio-Thoracic Surgery, and The Society of Thoracic Surgeons.

https://doi.org/10.1016/j.jtcvs.2020.11.001

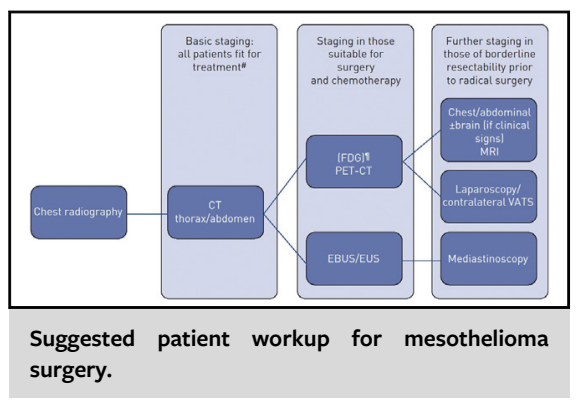

CENTRAL MESSAGE

Comparison of the most recent guidelines from major professional societies in America and Europe on the surgical management of malignant pleural mesothelioma reveals much agreement. Where differences do occur they reflect areas where good evidence is currently lacking.

the presence of fused pleural space) to confirm the presence of microscopic subpleural fat tissue invasion and to allow for adequate immunohistochemical analysis. Furthermore, there is a note on the importance of minimizing the number and size of incisions due to the risk of recurrence in the port-sites.

There is joint approval of the eighth edition of the tumor, node and metastasis (TNM) staging system (interpreted in Figure 1) and a recommendation to prospectively evaluate the importance of tumor volume or an approximation by tumor thickness. ${ }^{3}$ Both guidelines also recognize the value of modified Response Evaluation Criteria in Solid Tumours (RECIST 1.1) to determine tumor response after induction chemotherapy.

There is a tendency toward more complete staging for all patients in North America with the routine use of positron emission tomography-computed tomography (PET-CT) as well as specific recommendations for magnetic resonance imaging (MRI) with intravenous contrast (to assess the 


\begin{tabular}{|l|l|}
\hline T1 & Only ipsilateral parietal or visceral pleura \\
\hline T2 & Additional invasion of diaphragmatic muscle or lung parenchyma \\
\hline T3 & $\begin{array}{l}\text { In addition one or more of : } \\
\text { Invasion of chest wall +/- rib but resectable } \\
\text { Non-transmural pericardium } \\
\text { Mediastinal fat } \\
\text { Endothoracic fascia }\end{array}$ \\
\hline T4 & $\begin{array}{l}\text { In addition one or more of : } \\
\text { Multifocal chest wall invasion - unresectable } \\
\text { Transmural pericardial invasion +/- pericardial effusion } \\
\text { Mediastinal organs } \\
\text { Transdiaphragmatic invasion } \\
\text { Vertebrae or brachial plexus } \\
\text { Contralateral pleura }\end{array}$ \\
\hline N0 & No regional lymph node metastases \\
\hline N1 & $\begin{array}{l}\text { Metastasis to ipsilateral intrathoracic lymph nodes - including internal mammary, } \\
\text { intercostal and peridiaphragmatic }\end{array}$ \\
\hline N2 & Metastasis to contralateral intrathoracic lymph nodes + any supraclavicular nodes \\
\hline M0 & No distant metastases \\
\hline M1 & Any extrathoracic metastases \\
\hline
\end{tabular}

\begin{tabular}{|l|l|l|l|}
\hline STAGE & T & N & M \\
\hline IA & T1 & N0 & M0 \\
\hline IB & T2/T3 & N0 & M0 \\
\hline II & T1/T2 & N1 & M0 \\
\hline IIIA & T3 & N1 & M0 \\
\hline IIIB & T1/2/3 & N2 & M0 \\
\hline & T4 & N0/1/2 & M0 \\
\hline IV & Any T & Any N & M1 \\
\hline
\end{tabular}

FIGURE 1. The eighth edition of the tumor, node and metastasis (TNM) classification for malignant pleural mesothelioma—authors' interpretation.

subclavian vessels, chest wall, diaphragmatic, and mediastinal invasion), whereas the European approach appears to be more selective in recommending PET scan and MRI dependent on the eventual treatment destination. There is also agreement that findings on a PET-CT scan need to be confirmed by obtaining tissue, especially in surgical candidates when enlarged and/or PET avid mediastinal nodes are present. European and North American guidelines stress that endobronchial ultrasound has been found to have superior sensitivity and negative predictive value compared to mediastinoscopy for the assessment of nodal disease in MPM. Mediastinoscopy does, however, remain a valuable option for staging in the American protocol. There is agreement on the limited and selective use of staging laparoscopy and contralateral thoracoscopy when there is question about disease involvement in the peritoneum.

As expected, there is most divergence of opinion when the role of radical or "cytoreductive" surgery is considered. Radical surgery in MPM is defined as macroscopic complete resection (MCR), which can be achieved by extrapleural pneumonectomy (EPP) consisting of en bloc resection of the pleura, lung, pericardium and diaphragm, or (extended) pleurectomy/decortication (P/D), which includes resection of the total parietal and visceral pleura with or without part of the pericardium and diaphragm. Both procedures are combined with systematic mediastinal lymph nodes sampling or dissection for optimal staging.

The North American attitude is far more supportive of these procedures than that of the Europeans. In their recommendations, early-stage MPM should be treated by cytoreductive surgery as part of a multimodality approach. EPP remains a valuable option for patients who are good candidates with limited risk of major complications. The Europeans are more uncertain and recommend radical surgery in the context of clinical trials (of which there are few) or registries. Both European and North American guidelines recommend that cytoreductive surgery be performed in expert centres. 
The North American approach seems to mimic non-small cell lung cancer with primary surgery in stage I and stage II MPM followed by adjuvant chemotherapy. Induction chemotherapy is offered in stage III disease, particularly for patients with nodal involvement or unresectable disease, or as part of clinical trials. The European approach tends to favor upfront chemotherapy in all potentially resectable cases. The North American position is supported by published and inpress data showing disease progression in some patients who were treated first with neoadjuvant chemotherapy. ${ }^{4,5}$ To inform the debate on induction versus adjuvant chemotherapy, the EORTC 1205 trial (NCT02436733) currently randomizes patients with resectable MPM who all undergo extended $\mathrm{P} / \mathrm{D}$, according to a predefined surgical protocol, between induction and adjuvant chemotherapy. ${ }^{6}$ This randomized phase II trial is recruiting well.

Histological subtype is jointly recognized as an important selection criterion for radical surgery with both documents excluding known sarcomatoid MPM from major operations unless in clinical trials. However, the North Americans appear to be more aggressive toward biphasic MPM than the Europeans, particularly for epithelioid predominant biphasic tumors. Palliative treatment is recommended for sarcomatoid predominant tumors only. This differentiation may, however, be complicated by the difficulty to quantify the proportion of epithelioid and sarcomatoid component from the limited material obtained even by video-assisted thoracic surgery (VATS) pleural biopsy. ${ }^{7}$

The technique of radical surgery, not unsurprisingly, sees some areas of disagreement. Although there is an agreement on the objective of radical surgery, with regard to macroscopic complete resection (MCR), there is an underlying difference of opinion on its definition. The North American definition accepts a $1 \mathrm{~cm}$ area of residual disease, ${ }^{8}$ whereas in Europe, MCR means all visible tumor. There is an ongoing debate about whether all viable tumor equates to all visible tumor, in particular with regard to the management of the visceral pleura. ${ }^{9}$ The subplot surrounds a historical North American bias toward EPP over P/D, which many have traditionally considered to be a palliative debulking procedure; however, most North American centres have moved to $\mathrm{P} / \mathrm{D}$ in the past decade. The only randomized assessment of radical mesothelioma surgery, the MARS trial, ${ }^{10}$ originated from the UK; it was a feasibility study to assess the effect of adding EPP to chemotherapy in mediastinoscopy negative cases. A phase III study was not performed due to the difficulty in recruitment and lack of apparent benefit/evidence of potential harm in the early data. The small sample size of only 50 patients (24 EPP), however, has made interpretation problematic and, thus, it has been viewed through different eyes on either side of the Atlantic. In fact, the British Thoracic Society guidelines ${ }^{11}$ go so far as to outlaw the operation of EPP. The European approach is more cautious, advocating EPP only in specialized centres and advocating extended P/D as the approach of choice as part of multimodality treatment ${ }^{12}$ because of its potential lower postoperative morbidity and preservation of quality of life. There is, however, some irony in this recommendation as the operation of extended $\mathrm{P} / \mathrm{D}$ to achieve true MCR is a technically more demanding procedure and one more suited to higher volume specialist centres. Both guidelines recognize the limitations of the comparative studies between EPP and P/D due to their retrospective nature and the lack of tumor volume assessment (a marker of tumor stage) as a confounding factor.

The ASCO guidelines had a significant contribution from radiation oncologists which may explain their greater enthusiasm for radiotherapy in combination with radical surgery. Giving less weight to the attendant morbidity, they recommend strongly adjuvant high-dose hemithoracic radiotherapy, including pleural intensity-modulated radiotherapy after P/D. The European approach is far more guarded favoring a recommendation for more research on the subject only. The ultimate in aggressive therapy, the SMART protocol ${ }^{13}$ comprising induction hypofractionated hemithoracic radiotherapy followed by EPP, is suggested in the ASCO guidelines in the context of clinical trials, but not mentioned in the European document.

So much for the differences. What do the 2 documents share in their certainty?

There is surprising agreement regarding palliative surgery with both guidelines giving a role for VATS decortication, although the only randomized trial, MesoVATS, ${ }^{14}$ gave scant support such that this was another surgical procedure outlawed in the British guidelines. ${ }^{11}$ The North Americans are more adverse to indwelling pleural catheters as a bridge to radical surgery due to the risk of tumor progression along the track.

Europeans and North Americans also share the need for (1) better surgical descriptors to more clearly define the extent of resection that is required in MPM; (2) better definition of what constitute complete resection; (3) improved international collaboration to perform multicentre surgical trials with correlative studies particularly on circulating biomarkers; and (4) better clinical staging to adequately stratify patients to different treatment groups and be able to accurately compare outcomes. There is also a need for more translational research to understand the mechanisms of tumor progression and recurrence. ${ }^{15}$

With regard to radical surgery, the strength of the recommendations is typically not borne out by the strength of the evidence and neither group can be sure whether EPP or P/ $\mathrm{D}$ is the best treatment to offer for patients with nodepositive or biphasic MPM either before or after systemic therapy.

Although there is really no compelling evidence that radical surgery should be offered to patients with epithelioid, node-negative MPM, this is the group of patients 
who have the most to gain from surgery in the context of multimodality therapy. ${ }^{16}$ In this group of patients, most Europeans and North American will proceed with surgery. Some North Americans may proceed with EPP after radical hemithoracic radiotherapy, while the Europeans may choose lung-sparing P/D after induction chemotherapy.

Both groups await the results of the MARS2 trial, ${ }^{17}$ which is a phase III study of 328 patients with resectable MPM of any sub-type, comparing chemotherapy with or without additional P/D with the intent to achieve MCR. It is very near to completing recruitment and will, in a little over 2 years, address the question of whether P/D adds any survival benefit to systemic chemotherapy alone. The strength of the evidence may be persuasive, but its interpretation may lead to more questions than it does answers. A negative result may still leave burning questions about the possible superior role of EPP (especially from those who dismissed MARS1) and will leave tantalizing surgical questions over "what would have happened if the trial had been limited to the best actors with early stage epithelioid MPM?" Unfortunately, we may never get the opportunity to repeat MARS2 and yet, in best Hollywood blockbuster tradition, MARS3 - the sequel is already in the minds of existing trialists.

In the interim, after recruitment has finished but before the MARS2 study reports, what approach should surgeons take?

While awaiting the results of MARS2, we are justified in offering surgery as part of multimodality treatment to those with the best prognostic factors, ie, epithelioid with no clinical evidence of nodal disease. Those with nonepithelioid and/or nodal disease need to be assessed on a case-bycase basis in high-volume centres ideally as part of clinical trials or registries.

A transatlantic surgical consensus will not only be of academic benefit. Although the disease may be declining in incidence in North America, it remains at its peak in Western Europe and is predicted to move inexorably across the Globe from West to East. ${ }^{18}$ Therefore, lessons learnt in the West should lead us to produce agreed protocols which will be invaluable to inform the surgical decision-makers in Asia in the next decades of the twenty-first century.

\section{References}

1. Opitz I, Scherpereel A, Berghmans T, Psallidas I, Glatzer M, Rigau D, et al. ERS/ ESTS/EACTS/ESTRO guidelines for the management of malignant pleural mesothelioma. Eur J Cardiothorac Surg. 2020;58:1-24.
2. Kindler HL, Ismaila N, Armato SG III, Bueno R, Hesdorffer M, Jahan T, et al. Treatment of malignant pleural mesothelioma: American Society of Clinical Oncology clinical practice guideline. J Clin Oncol. 2018;36:1343-73.

3. Rusch VW, Chansky K, Kindler HL, Nowak AK, Pass HI, Rice DC, et al. The IASLC mesothelioma staging project: proposals for the M descriptors and for revision of the TNM stage groupings in the forthcoming (eighth) edition of the TNM classification for mesothelioma. J Thorac Oncol. 2016;11:2112-9.

4. Rimner A, Zauderer MG, Gomez DR, Adusumilli PS, Parhar PK, Wu AJ, et al. Phase II study of hemithoracic Intensity-Modulated Pleural Radiation Therapy (IMPRINT) as part of lung-sparing multimodality therapy in patients with malignant pleural mesothelioma. J Clin Oncol. 2016;34:2761-8.

5. Voigt SL, Raman V, Jawitz OK, Bishawi M, Yang CJ, Tong BC, et al. The role of neoadjuvant chemotherapy in patients with resectable malignant pleural mesothelioma-an institutional and national analysis. J Natl Cancer Inst 2020; djaa002.

6. Raskin J, Surmont V, Cornelissen R, Baas P, van Schil PEY, van Meerbeeck JP. A randomized phase II study of pleurectomy/decortication preceded or followed by (neo-)adjuvant chemotherapy in patients with early stage malignant pleural mesothelioma (EORTC 1205). Transl Lung Cancer Res. 2018;7:593-8.

7. Nicholson AG, Sauter JL, Nowak AK, Kindler HL, Gill RR, Remy-Jardin M, et al. EURACAN/IASLC proposals for updating the histologic classification of pleural mesothelioma: towards a more multidisciplinary approach. J Thorac Oncol. 2020;15:29-49.

8. Sugarbaker DJ, Wolf AS. Surgery for malignant pleural mesothelioma. Expert Rev Respir Med. 2010;4:363-72.

9. Friedberg JS, Culligan MJ, Tsao AS, Rusch V, Sepesi B, Pass HI, et al. A proposed system toward standardizing surgical-based treatments for malignant pleural mesothelioma, from the Joint National Cancer Institute-International Association for the Study of Lung Cancer-Mesothelioma Applied Research Foundation taskforce. J Thorac Oncol. 2019;14:1343-53.

10. Treasure T, Lang-Lazdunski L, Waller D, Bliss JM, Tan C, Entwisle J, et al. Extrapleural pneumonectomy versus no extrapleural pneumonectomy for patients with malignant pleural mesothelioma: clinical outcomes of the Mesothelioma and Radical Surgery (MARS) randomised feasibility study. Lancet Oncol. 2011;12:763-72.

11. Woolhouse I, Bishop L, Darlison L, De Fonseka D, Edey A, Edwards J, et al British Thoracic Society Guideline for the investigation and management of malignant pleural mesothelioma. Thorax. 2018;73:11-30.

12. Cao C, Tian D, Park J, Allan J, Pataky KA, Yan TD. A systematic review and meta-analysis of surgical treatments for malignant pleural mesothelioma. Lung Cancer. 2014;83:240-5.

13. de Perrot M, Wu L, Cabanero M, Perentes JY, McKee TD, Donahoe L, et al. Prognostic influence of tumor microenvironment after hypofractionated radiation and surgery for mesothelioma. J Thorac Cardiovasc Surg. 2020;159:2082-91.

14. Rintoul RC, Ritchie AJ, Edwards JG, Waller DA, Coonar AS, Bennett M, et al. Efficacy and cost of video-assisted thoracoscopic partial pleurectomy versus talc pleurodesis in patients with malignant pleural mesothelioma (MesoVATS): an open-label, randomised, controlled trial. Lancet. 2014;384:1118-27.

15. Tsao AS, Lindwasser OW, Adjei AA, Adusumilli PS, Beyers ML, Blumenthal GM, et al. Current and future management of malignant mesothelioma: a consensus report from the National Cancer Institute Thoracic Malignancy Steering Committee, International Association for the Study of Lung Cancer, and Mesothelioma Applied Research Foundation. J Thorac Oncol. 2018;13:1655-67.

16. Nelson DB, Rice DC, Niu J, Atay S, Vaporciyan AA, Antonoff M, et al. Longterm survival outcomes of cancer-directed surgery for malignant pleural mesothelioma: propensity score matching analysis. J Clin Oncol. 2017;35:3354-62.

17. Lim E. A feasibility study comparing extended pleurectomy/decortication versus no pleurectomy/decortication in the multimodality management of patients with malignant pleural mesothelioma: the MARS2 study. Lung Cancer. 2016;91:S71.

18. Kazan-Allen L. Asbestos and mesothelioma: worldwide trends. Lung Cancer. 2005;49:S3-8 